Service social

\title{
Les multiples facettes du rapport à l'autre
}

\section{Daniel Turcotte}

Volume 43, numéro 2, 1994

Enfants, parents, intervenants

URI : https://id.erudit.org/iderudit/706653ar

DOI : https://doi.org/10.7202/706653ar

Aller au sommaire du numéro

Éditeur(s)

École de service social de l'Université Laval

ISSN

1708-1734 (numérique)

Découvrir la revue

Citer ce document

Turcotte, D. (1994). Les multiples facettes du rapport à l'autre. Service social, 43(2), 3-6. https://doi.org/10.7202/706653ar d'utilisation que vous pouvez consulter en ligne.

https://apropos.erudit.org/fr/usagers/politique-dutilisation/ 


\section{AVANT.PROPOS}

\section{Les multiples facettes du rapport à l'autre}

Dans la planification de la revue, le présent numéro avait été prévu comme un numéro sans thème portant sur des sujets diversifiés qui n'avaient pas nécessairement de lien. Or, à la lecture des articles, un thème est apparu en filigrane dans tous les textes: le rapport à l'autre. Rapport des personnes entre elles, notamment entre les enfants, les parents et les intervenants, mais également rapport entre les personnes et les organisations qui gravitent autour d'elles. Ce rapport peut se matérialiser par l'influence que les parents exercent sur les enfants, par les transactions que les familles engagent avec les organisations ou à travers les relations que les intervenants et les entraidants développent. Comme l'illustrent les différents textes de ce numéro, les multiples facettes de ce rapport à l'autre dénotent un élargissement des façons d'aborder l'aide aux enfants et aux familles, une affirmation de la nécessité de s'appuyer sur les compétences des personnes et une reconnaissance de la nécessité de mettre à contribution le plus large éventail possible de ressources dans une dynamique de complémentarité.

Dans un contexte où les problèmes psychosociaux sont de plus en plus complexes et les ressources institutionnelles de plus en plus limitées, il est indispensable de pousser plus à fond notre compréhension des difficultés que rencontrent les enfants, les parents et les intervenants (professionnels, entraidants et bénévoles), afin d'élaborer des façons de faire susceptibles de faciliter les transitions qui se 
produisent au cours de la vie et de favoriser le développement optimal des personnes. C'est à cette tâche que s'emploient les auteurs des textes de ce numéro.

Dans le premier article, un collectif d'auteurs, regroupés dans le cadre de la création d'un partenariat entre intervenants et chercheurs, présente une recension des écrits sur le profil psychosocial des différents acteurs associés à l'abus sexuel. Après avoir relevé la difficulté que pose la définition même de la notion d'abus sexuel, les auteurs traitent des séquelles chez les victimes, des caractéristiques des pères abuseurs et des mères ainsi que des modes d'organisation des relations au sein des familles où se produit l'abus. Ils complètent l'article en soulignant la portée et les limites des connaissances actuelles sur cette problématique et en indiquant trois préoccupations qui devraient guider les recherches descriptives dans ce domaine.

Partant du constat que la séparation conjugale peut s'accompagner de conséquences négatives pour l'enfant et que ces conséquences peuvent être atténuées par une intervention appropriée auprès des parents, Lemieux et Cloutier ont mis sur pied un programme d'intervention, s'adressant aux parents gardiens, dont I'objectif est de favoriser l'adaptation de l'enfant à la séparation. Leur article présente les résultats de l'évaluation de ce programme, résultats qui révèlent, notamment, une amélioration de la relation parentenfant et une modification des perceptions des parents de l'adaptation de l'enfant. À la lumière de leurs observations, ils avancent l'idée que la participation du parent non gardien et des enfants pourrait améliorer le potentiel d'un tel programme.

Dans la continuité du rapport entre les parents et l'enfant, Trudelle et Montambault présentent les résultats d'une recherche sur le sentiment de compétence parentale de pères et de mères d'enfants d'âge préscolaire. Globalement, ils observent que les variables associées aux sentiments de compétence, de satisfaction et d'efficacité varient selon le sexe du parent. Ils concluent en affirmant la nécessité de poursuivre des recherches sensibles à la complexité de la réalité familiale et axées sur la compréhension de la façon dont le sentiment de compétence des parents se façonne au fil des événements et du temps.

L'article de Deslandes et Royer traite également du rapport parent-enfant, mais cette fois sous l'angle de l'influence des parents sur la réussite scolaire de leur enfant. S'appuyant sur une recension des recherches sur l'influence du milieu familial et des liens familleécole sur la réussite scolaire, ils proposent un nouveau cadre conceptuel pour l'étude des interactions famille-école: le modèle révisé de 
I'influence partagée, modèle qui intègre l'influence du style parental et des pratiques parentales dans l'analyse du partenariat famille-école.

Poulin, Beaudoin et Turgeon-Krawczuk abordent le rapport entre les parents et la maladie de leur enfant, à travers I'utilisation de I'informatique. Ils présentent les résultats d'une recherche portant sur I'utilisation par les parents du logiciel interactif Vie familiale et impact de la maladie. Leur expérimentation auprès de quarante parents fait ressortir des attitudes très favorables à l'utilisation de l'informatique dans une perspective d'intervention: les parents jugent le contenu du logiciel pertinent par rapport à leurs problèmes et ils y voient une utilité comme déclencheur de réflexion et d'échanges.

Le rapport à l'autre ne s'inscrit pas nécessairement dans le cadre $d^{\prime}$ 'une relation interpersonnelle ; il peut également prendre forme dans le rapport aux institutions. C'est là le sujet du texte de Fortin et Legault qui porte sur les résultats d'une recherche exploratoiredescriptive conduite auprès de familles immigrantes et $d^{\prime}$ intervenants sur les difficultés rencontrées par ces familles et sur les modalités de résolution de problèmes qu'elles jugent prioritaires. Outre la diversité des difficultés rencontrées et l'écart entre la perception des familles et celle des intervenants quant à la hiérarchisation de ces difficultés, les résultats indiquent que les familles immigrantes préfèrent recourir au réseau formel des services lorsqu'elles font face à des problèmes sociaux, mais elles privilégient le réseau informel pour leurs difficultés d'ordre culturel. Pour faciliter l'intégration des familles immigrantes, les auteurs suggèrent de mettre en place un service basé sur le jumelage de ces nouvelles familles avec des familles de la société d'accueil.

Si pendant longtemps les organismes bénévoles ont pu s'enorgueillir du caractère informel de leur fonctionnement, actuellement la situation serait différente. Selon Robichaud, les groupes bénévoles, en raison des nouvelles exigences de l'État, se voient maintenant forcés de situer leurs actions dans des cadres structurels qui s'apparentent à des quasi-appareils. Si cette structuration plus formelle des rapports et des pratiques atténue le champ de la spontanéité, elle n'entraîne pas nécessairement une perte d'attrait pour le travail bénévole. En dépit des contraintes qui pèsent sur elles, les personnes bénévoles parviennent à préserver une zone $\mathrm{d}^{\prime}$ autonomie qui les conduit à poursuivre leur engagement. Cependant, l'auteure s'interroge en conclusion sur l'impact éventuel, à plus long terme, du mouvement d'institutionnalisation du bénévolat dans lequel l'État est engagé.

II y a toujours un risque qu'un trop grand formalisme constitue un frein à la créativité et fasse obstacle à l'émergence de pratiques 
innovatrices comme celle que présente le texte de Thivierge qui porte sur l'intervention de groupe auprès de la population itinérante de Montréal. Récipiendaire, en 1994, du prix d'excellence du Fonds Simone-Paré, pour l'originalité de son intervention et la ferveur de son engagement professionnel, François Thivierge décrit dans son article l'itinéraire d'un groupe de personnes qui ont cheminé, à travers l'entraide, vers I'autonomie et la dignité.

Qu'ils concernent les enfants, les parents, les entraidants ou les intervenants, les textes de ce numéro présentent un reflet de la diversité des perspectives qui orientent l'intervention sociale. II reste à espérer que chaque lectrice, chaque lecteur pourra y trouver une idée qui saura inspirer sa façon d'entrer en rapport avec les autres.

Daniel TURCOTTE 